\title{
$A b$ initio Computational Study of Quantum Plasmons in Graphene Nanoflakes
}

\author{
David Zs. Manrique ${ }^{1}$ and Nicolae C. Panoiu ${ }^{1}$
}

\begin{abstract}
We investigate the potential merit in using nanometer-sized graphene flakes as building blocks of twodimensional (2D) quantum metamaterials. The choice of the building blocks is crucial to the design of quantum metamaterials with desired properties, graphene nano-structures being promising candidates towards this end. Thus, they can be grown either by bottom-up chemical synthesis or top-down electronbeam patterning in various shapes, densities, topology, and size, down to the molecular scale. We show that this versatility provides a wide range of parameters to tune the optical properties of graphene-based $2 \mathrm{D}$ quantum metamaterials.
\end{abstract}

\section{INTRODUCTION}

Graphene is beginning to play a crucial role in the emerging area of quantum plasmonics, mainly due to its strong interaction with light and peculiar electron dynamics. The surface plasmons (collective charge oscillations) in graphene [1], [2] are much more confined and low-loss than the conventional plasmonic materials, such as metallic nanoparticles and nanowires. Inheriting these remarkable properties from the extended sheet, graphene nano-flakes (GNFs) also exist in stable form, either grown by using bottom-up chemical synthesis methods or tailored by top-down electron-beam techniques. Recent growth of nanometer-sized GNFs has paved a tantalizing route towards systematically engineering GNFs to manipulate the optical near-field at the nano-scale and quantum level. The surface plasmons in these zerodimensional (0D) GNFs [3] are even more confined than in extended graphene sheets and can be strikingly different from those of metallic nanoparticles. In particular, OD GNFs support edge plasmons, this reduced dimensionality having fundamental implications on their physical properties. Equally important, by using nanometer-sized GNFs, the plasmon resonance frequency could reach the visible-light spectrum region, which otherwise can be difficult to achieve by using regular resonant plasmonic structures.

In this study, by using time-dependent density-functional theory (TD-DFT) and quantum chemistry computations [4], [5], we show that GNFs support collective charge oscillations, called quantum plasmons, whose physical properties exhibit strong dependence on the shape, size, and topology of the GNF. For example, we show that increasing the size of the nanoflakes by a few nanometers results in a redshift of the order of $1 \mathrm{eV}$ of the plasmon frequency.

This work was supported by the European Research Council / ERC Grant Agreement no. ERC-2014-CoG-648328.

${ }^{1}$ The authors are with the Department of Electronic and Electrical Engineering, University College London, Torrington Place, London WC1E 7JE, UK. d.manrique@ucl.ac.uk; n.panoiu@ucl.ac.uk

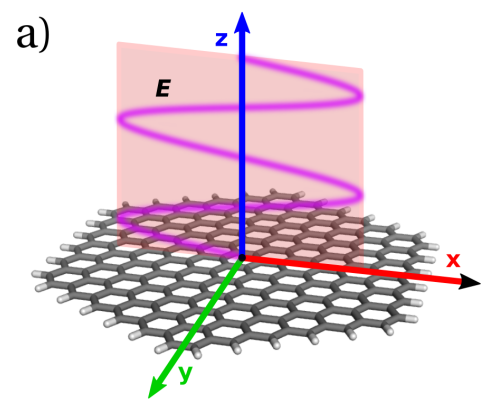

b)

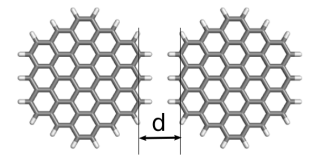

Fig. 1. a) Illustration of geometrical setup in the calculations. The driving light wave impinges perpendicularly onto the plane of the graphene nanoflakes and is modeled as a spatially constant electric field along the $x$-axis. b) Schematics of dimers made of hexagonal and triangular GNFs.

\section{RESUlTS AND Discussion}

We have first computed the ground state of the GNFs presented in Fig. 1, namely hexagonal and triangular GNFs as well as dimers made of such GNFs. Then we have performed TD-DFT simulations to determine the system dynamics and, implicitly, the optical absorption spectra. The computational domain was defined by encapsulating each atom in a sphere with a radius of $8 \AA$ and a uniform mesh grid of $0.3 \AA$. A Fermi-Dirac type function has been chosen to smear the Fermi surface and the electronic temperature was set to be $\sim 10 \mathrm{~K}(0.001 \mathrm{eV})$. We have used a delta-function time-dependent potential, $v(\vec{r}, t)=-A \hbar k_{0} x \delta(t)$, where $\hbar k_{0}$ is the photon momentum, and $A$, an adjustable constant, has been chosen to be very small so as to ensure that the response of the system is linear. The TD-DFT equation was subsequently solved in the time and space domains, and the corresponding Kohn-Sham wave functions were then propagated for a relatively long time by using approximately enforced time-reversal symmetry methods [4].

We considered first a single GNF of hexagonal shape, the size of the flake being $0.75 \mathrm{~nm}$. Among other features, the corresponding optical absorption spectrum, presented in Fig. 2(a), displays two resonances at about $3.5 \mathrm{eV}$ and $6 \mathrm{eV}$. In order to understand the physical nature of these resonances, we investigated the corresponding time evolution of the charge density when the system is driven at these particular frequencies. For this, we gradually (exponentially) turned on a sine-shape continuous-wave electric field excitation whose frequency, $\omega$, matches that of the spectral peak under investigation and recorded the steady-state evolution of the charge density. The results of these calculations, 

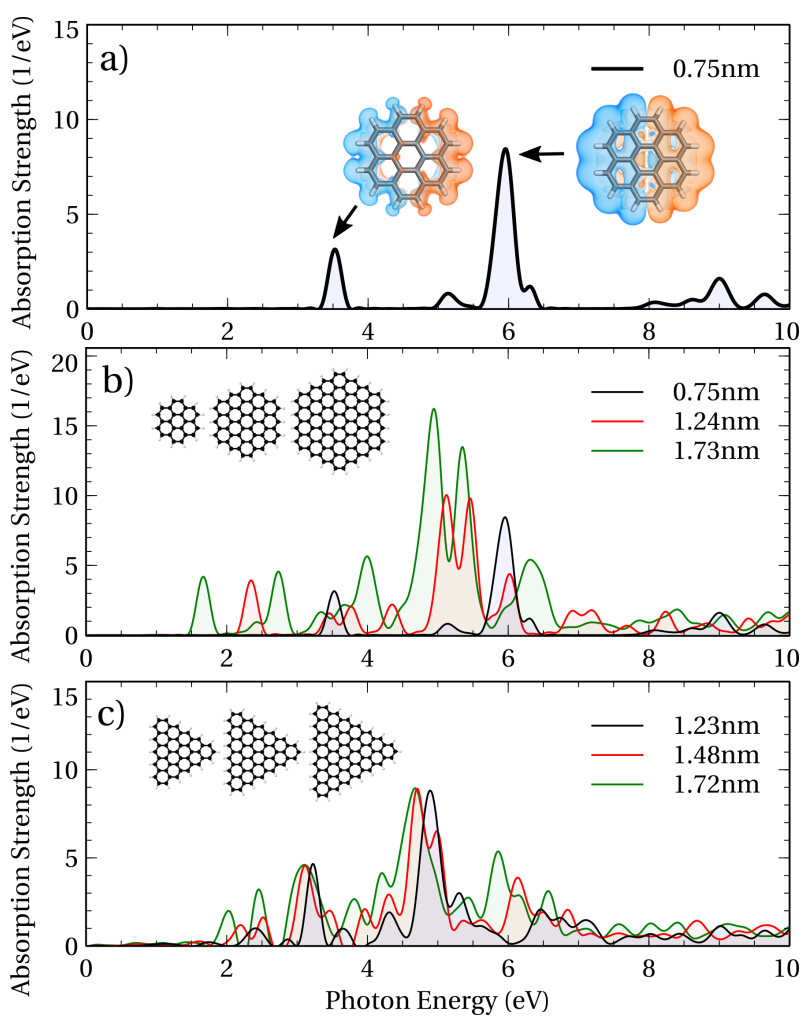

Fig. 2. a) Absorption spectra of a hexagonal GNF. The inset illustrates the maximum charge density change over a driving period across the molecule at the marked resonance energies. b) Absorption spectra of hexagonal GNFs with increasing size, shown in the inset. The legend indicates the diameter of the hexagons. c) Absorption spectra of triangular GNFs with increasing size, shown in the inset. The legend indicates the side length of the triangles.

namely the charge densities corresponding to the two main absorption peaks, are shown as insets in Fig. 2(a). The collective nature of the charge density oscillations illustrated by these plots suggests that these are quantum plasmons.

We also investigated the dependence of the properties of quantum plasmons on the size and shape of GNFs, by considering hexagonal and triangular flakes with increasing size. The main conclusion of this analysis, illustrated in Figs. 2(b) and 2(c), is that the energy of quantum plasmons decreases with the size of the GNF, a result that is valid in the classical context, too. This size dependence of the plasmon energy is particularly strong in the case of hexagonal GNF, the energy of the fundamental plasmon varying from $3.5 \mathrm{eV}$ to $1.75 \mathrm{eV}$ when the flake size increases from $0.75 \mathrm{~nm}$ to $1.73 \mathrm{~nm}$. By contrast, the spectra of triangular GNFs are affected less by the variation of the size of the flake.

Finally, we have investigated the interaction between quantum plasmons of GNFs and how this interaction depends on the system parameters. For this, we considered dimers made of hexagonal and triangular GNFs separated by a variable distance, $d$, and in both cases determined the optical absorption spectra. The results of these calculations, presented in Fig. 3, show that even if the GNFs interact via just a few atoms a frequency shift of the optical spectra (more pronounced in the case of hexagonal GNF dimers)
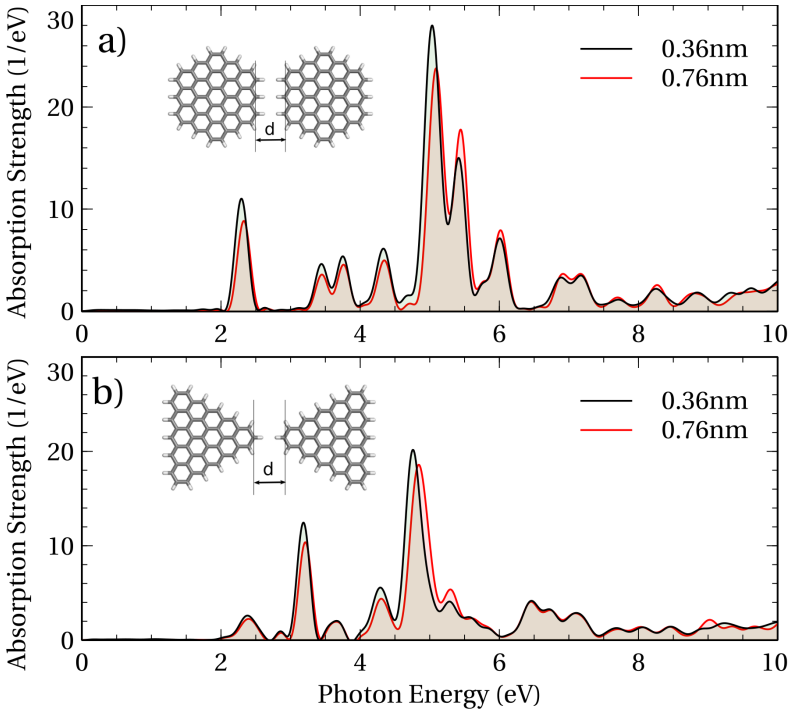

Fig. 3. a) Absorption spectra of a dimer made of hexagonal GNFs with $1.24 \mathrm{~nm}$ diameter. b) Absorption spectra of a dimer made of triangular GNFs with $1.23 \mathrm{~nm}$ side length. The legends show the distance $d$, as illustrated in the insets, where $d=0.36 \mathrm{~nm}$ corresponds to $0.15 \mathrm{~nm} \mathrm{H}-\mathrm{H}$ distance.

can be observed. More specifically, the interaction between the GNFs splits the plasmon spectral peaks, which allows a fine-tuning of the corresponding frequencies by changing the separation distance or charge doping. For small sizes of the GNFs, the collective electron flow is controlled by the valence orbital structure rather than classical plasmonic oscillation of free electrons, which allows atomically precise design of the electronic motion pattern. Such precisely designed nano-sized graphene building blocks can be used for ultra-sensitive single molecular detection or molecular scale plasmonic electric field enhancement.

\section{CONCLUSIONS}

In conclusion, we have demonstrated that molecular excitations on GNF can be viewed as quantum plasmons. Our study revealed that the physical properties of these quantum plasmons depend strongly on the material and geometrical parameters of the GNFs. We also found that in a dimer configuration the plasmonic frequencies can be slightly shifted with separation controlled intermolecular interactions between multiple GNFs.

\section{REFERENCES}

[1] K. Novoselov, A. Geim, S. Morozov, D. Jiang, Y. Zhang, S. Dubonos, I. Grigorieva, and A. Firsov, Electric field effect in atomically thin carbon films", Science, vol. 306, p. 666, 2004.

[2] A. Castro Neto, F. Guinea, N. Peres, K. Novoselov, and A. Geim, "The electronic properties of graphene", Reviews of Modern Physics, vol. 81, p. 109, 2009.

[3] S. Thongrattanasiri, A. Manjavacas, and F. Javier García de Abajo, "Quantum Finite-Size Effects in Graphene Plasmons", ACS Nano, vol. 6, p. 1766, 2012.

[4] A. Castro, H. Appel, M. Oliveira, C. A. Rozzi, X. Andrade, F. Lorenzen, M. A. L. Marques, E. Gross, and A. Rubio, "Octopus: a Tool for the Application of Time-Dependent Density Functional Theory", Physica Status Solidi B, vol. 243, p. 2465, 2006.

[5] E. Runge and E. K. U. Gross, "Density-Functional Theory for TimeDependent Systems", Physical Review Letters, vol. 52, p. 997, 1984. 